\title{
CDISC SEND Planned Pharmacologic Target Mode of Action Response Terminology
}

National Cancer Institute

\section{Source}

National Cancer Institute. CDISC SEND Planned Pharmacologic Target Mode of Action

Response Terminology. NCI Thesaurus. Code C154684.

Terminology associated with the planned pharmacologic target mode of action response codelist of the Clinical Data Interchange Standards Consortium (CDISC) Standard for the Exchange of Non-clinical Data (SEND). 